\title{
Correlation and Path Analysis of Kernel Yield and its Components in Groundnut (Arachis hypogaea L.)
}

\section{A. Trivikrama Reddy ${ }^{*}$, M. Reddi Sekhar ${ }^{2}$, A. Vijayabharathi ${ }^{3}$, T. Lakshmi Pathy ${ }^{4}$,} G. Lakshmikantha Reddy ${ }^{2}$ and V. Jayalakshmi ${ }^{1}$

${ }^{1}$ Regional Agricultural Research Station, Nandyal - 518502, ANGRAU, Andhra Pradesh, India

${ }^{2}$ Department of Genetics and Plant Breeding, S.V. Agricultural College, Tirupati - 517502, Andhra Pradesh, India

${ }^{3}$ Department of Genetics and Plant Breeding, University of Agricultural Sciences, GKVK, Bangalore - 560065, Karnataka, India

${ }^{4}$ ICAR-Sugarcane Breeding Institute, Coimbatore, Tamil Nadu - 641007, India

*Corresponding author

\section{A B S T R A C T}

\section{Keywords}

Groundnut, Correlation and path analysis.

\section{Article Info}

Accepted:

04 October 2017

Available Online:

10 December 2017
Character association and path analysis were carried out for kernel yield and its component characters in six parents and their $15 \mathrm{~F}_{1}$ crosses in groundnut. The genotypic correlation coefficients were observed to be relatively of higher magnitude than the corresponding genotypic and phenotypic correlation coefficients indicating strong inherent association between the characters. Kernel yield per plant possessed significant positive association with pod yield per plant, mature pods per plant, 100-kernel weight, pods per plant, pegs per plant, harvest index and shelling percentage at both the genotypic and phenotypic levels. Path analysis also revealed the importance of these traits as they had direct effects on kernel yield. Therefore, selection based on these characters will lead to simultaneous improvement in kernel yield in groundnut.

\section{Introduction}

Biometrical procedures such as correlations and path analysis establish the extent of association between yield and its attributes, so that these yield components may form additional criteria for selection in breeding programmes. Yield is a complex entity and depends on the expression of a number of traits known as yield components. The knowledge of association of yield components with yield components with yield and among themselves will be helpful in the improvement of a complex character like yield for which direct selection is not very effective. The correlations with path analysis would give a better appreciation of cause and effect relationship between different pairs of characters.

\section{Materials and Methods}

The materials for the study comprised of six parents and their $15 \mathrm{~F}_{1}$ crosses obtained in a 
half diallel method were raised in a RBD replicated thrice with a spacing of $30 \times 10 \mathrm{~cm}$ at S.V. Agricultural College, Tirupati, Andhra Pradesh. Data were recorded on a ten randomly selected plants in each entry of each replication for thirteen quantitative traits viz., branches per plant, days to $50 \%$ flowering, days to maturity, plant height, pegs per plant, pods per plant, mature pods per plant, per cent pod set, harvest index, shelling percentage, 100-kernel weight, pod yield per plant and kernel yield per plant. Phenotypic and genotypic correlations were worked out using the formula suggested by Jhonson et al., (1955). The path coefficient analysis was carried out according to Dewey and Lu (1959).

\section{Results and Discussion}

The differences among the genotypes were significant for all the characters studied. Genotypic correlations were of higher magnitude than the phenotype correlations (Table 1) indicating strong inherent association between the two corresponding characters and selection for these characters might be rewarding.

The characters pod yield per plant, mature pods per plant, 100-kernel weight, pods per plant, pegs per plant, harvest index and shelling percentage had highly significant and positive association with kernel yield per plant in the and also exhibited significant positive inter-correlations among themselves.

These results indicated that selection for these characters would simultaneously bring improvement in the associated characters and finally in yield (Mahalakshmi et al., 2005, Patil et al., 2006, Sumathi and Muralidharan et al., 2007, Sawargaonkar et al., 2010, Shukla et al., 2014, Shashi Kumara et al., 2015, Jain et al., 2016, Ramakrishnan et al., 2017 and Rajarathinam et al., 2017). Kernel yield also had non-significant positive association with plant height, per cent pod set, days to $50 \%$ flowering and days to maturity. These observations were in conformity with the reports of Alam et al., (1985) for days to maturity and plant height; Rasheed et al., (2015) for plant height.

Contrary to these findings, Dhaliwal et al., (2010), Sawargaonkar et al., (2010) and Vange and Maga (2014) noticed significant positive association of plant height, days to $50 \%$ flowering, days to maturity on kernel yield in groundnut.

Kernel yield had non-significant negative association with branches per plant and it was in conformity with the findings of Antony et al., (2000). Contrary to this, significant positive association between kernel yield per plant and branches per plant was reported by Khanpara et al., (2010), Vange and Maga (2014) and Prabhu et al., (2016) in groundnut.

The phenotypic correlation between kernel yield and its component characters were partitioned in to the direct and indirect components (Table 2).

The pod yield exerted the highest positive direct effect on kernel yield followed by shelling percentage and pegs per plant. Similar results were reported by Shukla et al., (2014) for shelling percentage and Vijayasekhar (2002) for pod yield and pegs per plant; for pod yield by Shoba et al., (2012), Thirumala Rao, (2016) and Rajarathinam et al., (2017); for pod yield per plant and shelling percentage by Pavan Kumar et al., (2014). Through pegs per plant characters such as days to maturity, pods per plant, mature pods per plant, pod yield per plant exhibited negative indirect effect on kernel yield per plant, whereas shelling percentage displayed positive indirect influence. 
Table.1 Phenotypic $\left(r_{\mathrm{p}}\right)$ and genotypic $\left(r_{\mathrm{g}}\right)$ correlation coefficients between kernel yield and its components in groundnut

\begin{tabular}{|c|c|c|c|c|c|c|c|c|c|c|c|c|c|}
\hline Character & & $\mathbf{X}_{2}$ & $\mathbf{X}_{3}$ & $\mathbf{X}_{4}$ & $\mathbf{X}_{5}$ & $\mathbf{X}_{6}$ & $\mathbf{X}_{7}$ & $\mathbf{X}_{8}$ & $X_{9}$ & $\mathbf{X}_{10}$ & $X_{11}$ & $X_{12}$ & $\mathbf{X}_{13}$ \\
\hline \multirow{2}{*}{$\mathbf{X}_{1}$} & $r_{\mathrm{p}}$ & -0.289 & -0.085 & -0.324 & 0.134 & 0.223 & 0.136 & 0.257 & -0.030 & 0.037 & 0.082 & -0.043 & -0.035 \\
\hline & $r_{\mathrm{g}}$ & $-0.634 * *$ & -0.073 & -0.387 & 0.222 & 0.346 & 0.201 & 0.364 & -0.227 & 0.019 & 0.119 & -0.193 & -0.137 \\
\hline \multirow{2}{*}{$\mathbf{X}_{2}$} & $r_{\mathrm{p}}$ & & 0.310 & -0.083 & 0.051 & -0.018 & 0.045 & -0.120 & 0.305 & -0.058 & -0.214 & 0.151 & 0.093 \\
\hline & $r_{\mathrm{g}}$ & & $0.504 *$ & -0.350 & -0.048 & -0.243 & -0.087 & -0.397 & $0.555 * *$ & -0.015 & -0.323 & 0.036 & -0.002 \\
\hline \multirow{2}{*}{$\mathbf{X}_{3}$} & $r_{\mathrm{p}}$ & & & -0.429 & 0.307 & 0.125 & 0.076 & -0.288 & 0.359 & -0.105 & -0.058 & 0.104 & 0.082 \\
\hline & $r_{\mathrm{g}}$ & & & $-0.624 * *$ & $0.439 *$ & 0.226 & 0.232 & -0.329 & $0.519 *$ & -0.204 & -0.135 & 0.181 & 0.103 \\
\hline \multirow{2}{*}{$\mathbf{X}_{4}$} & $r_{\mathrm{p}}$ & & & & 0.220 & 0.349 & 0.368 & 0.259 & -0.354 & -0.074 & -0.064 & 0.193 & 0.214 \\
\hline & $r_{\mathrm{g}}$ & & & & 0.137 & 0.332 & 0.383 & 0.397 & $-0.482 *$ & -0.076 & 0.032 & 0.394 & 0.320 \\
\hline \multirow{2}{*}{$\mathbf{X}_{5}$} & $r_{\mathrm{p}}$ & & & & & $0.882 * *$ & $0.813 * *$ & -0.032 & -0.037 & -0.386 & 0.209 & $0.533^{*}$ & 0.415 \\
\hline & $r_{\mathrm{g}}$ & & & & & $0.891 * *$ & $0.847 * *$ & -0.030 & 0.003 & $-0.477 * *$ & 0.122 & 0.618 & $0.445^{*}$ \\
\hline \multirow{2}{*}{$\mathbf{X}_{6}$} & $r_{\mathrm{p}}$ & & & & & & $0.837 * *$ & 0.432 & -0.084 & -0.224 & -0.002 & $0.507 *$ & $0.453 *$ \\
\hline & $r_{\mathrm{g}}$ & & & & & & $0.858 * *$ & 0.423 & -0.108 & -0.269 & 0.064 & $0.597 *$ & $0.479 *$ \\
\hline \multirow{2}{*}{$\mathbf{X}_{7}$} & $r_{\mathrm{p}}$ & & & & & & & 0.215 & 0.015 & -0.111 & 0.172 & $0.631 * *$ & $0.593 * *$ \\
\hline & $r_{\mathrm{g}}$ & & & & & & & 0.183 & 0.012 & -0.100 & 0.318 & $0.704 * *$ & $0.637 * *$ \\
\hline \multirow{2}{*}{$\mathbf{X}_{8}$} & $r_{\mathrm{p}}$ & & & & & & & & -0.102 & 0.302 & -0.055 & 0.066 & 0.188 \\
\hline & $r_{\mathrm{g}}$ & & & & & & & & -0.187 & 0.422 & -0.096 & 0.092 & 0.202 \\
\hline \multirow{2}{*}{$X_{9}$} & $r_{\mathrm{p}}$ & & & & & & & & & 0.251 & 0.343 & 0.255 & 0.340 \\
\hline & $r_{\mathrm{g}}$ & & & & & & & & & $0.535^{*}$ & 0.404 & 0.310 & 0.381 \\
\hline \multirow{2}{*}{$\mathbf{X}_{10}$} & $r_{\mathrm{p}}$ & & & & & & & & & & 0.297 & 0.003 & 0.322 \\
\hline & $r_{\mathrm{g}}$ & & & & & & & & & & 0.387 & 0.215 & $0.463 *$ \\
\hline \multirow{2}{*}{$\mathbf{X}_{11}$} & $r_{\mathrm{p}}$ & & & & & & & & & & & $0.452 *$ & $0.517 *$ \\
\hline & $r_{\mathrm{g}}$ & & & & & & & & & & & $0.604 * *$ & $0.612 * *$ \\
\hline \multirow{2}{*}{$X_{12}$} & $r_{\mathrm{p}}$ & & & & & & & & & & & & $0.906 * *$ \\
\hline & $r_{\mathrm{g}}$ & & & & & & & & & & & & $0.979 * *$ \\
\hline
\end{tabular}

* Significant at $P=0.05 \quad * *$ Significant at $P=0.01$

$\mathbf{X}_{\mathbf{1}}$ - Branches per plant

$\mathbf{X}_{\mathbf{2}}$ - Days to $50 \%$ flowering

$\mathbf{X}_{\mathbf{3}}$ - Days to maturity

$\mathbf{X}_{4}$ - Plant height $(\mathrm{cm})$
$\mathbf{X}_{\mathbf{5}}$ - Pegs per plant

$\mathbf{X}_{6}$ - Pods per plant

$\mathbf{X}_{7}$ - Mature pods per plant

$\mathbf{X}_{\mathbf{8}}$ - Per cent pod set
$\mathbf{X}_{\mathbf{9}}$ - Harvest index

$\mathbf{X}_{\mathbf{1 0}}$ - Shelling percentage

$\mathbf{X}_{11}$ - 100-Kernel weight

$\mathbf{X}_{12}$ - Pod yield per plant (g)
$\mathbf{X}_{13}$ - Kernel yield per plant $(\mathrm{g})$ 
Table.2 Phenotypic $(P)$ and genotypic $(G)$ path coefficients between kernel yield and its components in groundnut

\begin{tabular}{|c|c|c|c|c|c|c|c|c|c|c|c|c|c|c|}
\hline Character & & X1 & X2 & $\mathbf{X 3}$ & X4 & X5 & X6 & $\mathbf{X 7}$ & X8 & X9 & $\mathrm{X} 10$ & X11 & X12 & $r^{2}$ \\
\hline X1 & $G$ & 0.072 & -0.008 & 0.000 & 0.015 & -0.138 & 0.303 & -0.018 & -0.204 & 0.044 & 0.011 & -0.018 & -0.195 & -0.137 \\
\hline $\mathrm{X} 2$ & $G$ & -0.046 & 0.013 & 0.002 & 0.013 & 0.030 & -0.213 & 0.008 & 0.222 & -0.108 & -0.008 & 0.048 & 0.036 & -0.002 \\
\hline \multirow[t]{2}{*}{ X3 } & $P$ & 0.001 & -0.011 & 0.046 & -0.044 & 0.046 & -0.013 & 0.002 & -0.022 & 0.029 & -0.033 & -0.002 & 0.084 & 0.010 \\
\hline & $G$ & -0.005 & 0.007 & 0.004 & 0.024 & -0.273 & 0.198 & -0.021 & 0.184 & -0.101 & -0.116 & 0.020 & 183 & .103 \\
\hline \multirow[t]{2}{*}{ X5 } & $P$ & -0.001 & -0.002 & 0.014 & 0.022 & 0.149 & -0.089 & 0.018 & -0.002 & -0.003 & -0.122 & 0.001 & 0.428 & 0.294 \\
\hline & $G$ & 0.016 & -0.001 & 0.002 & -0.005 & -0.621 & 0.779 & -0.077 & 0.017 & -0.001 & -0.271 & -0.018 & 0.626 & $0.445 *$ \\
\hline \multirow[t]{2}{*}{ X6 } & $P$ & -0.001 & 0.001 & 0.006 & 0.036 & 0.132 & -0.101 & 0.018 & 0.033 & -0.007 & -0.071 & 0.000 & 0.407 & 0.354 \\
\hline & $G$ & 0.025 & -0.003 & 0.001 & -0.013 & -0.554 & 0.874 & -0.078 & -0.236 & 0.021 & -0.153 & -0.010 & 0.604 & 0.479* \\
\hline \multirow[t]{2}{*}{$\mathrm{X} 7$} & $P$ & -0.001 & -0.002 & 0.004 & 0.038 & 0.121 & -0.084 & 0.022 & 0.017 & 0.001 & -0.035 & 0.005 & 0.507 & $0.467 *$ \\
\hline & $G$ & 0.015 & -0.001 & 0.001 & -0.015 & -0.526 & 0.750 & -0.091 & -0.102 & -0.002 & -0.057 & -0.048 & 0.713 & $0.637 * *$ \\
\hline \multirow[t]{2}{*}{$\mathbf{X 1 0}$} & $P$ & 0.000 & 0.002 & -0.005 & -0.008 & -0.058 & 0.023 & -0.002 & 0.023 & 0.020 & 0.315 & 0.009 & 0.002 & 0.065 \\
\hline & $G$ & 0.001 & 0.000 & -0.001 & 0.003 & 0.296 & -0.235 & 0.009 & -0.236 & -0.104 & 0.569 & -0.058 & 0.218 & 0.463* \\
\hline \multirow[t]{2}{*}{ X11 } & $P$ & -0.001 & 0.008 & -0.003 & -0.007 & 0.004 & 0.000 & 0.004 & -0.004 & 0.027 & 0.094 & 0.031 & 0.363 & 0.208 \\
\hline & $G$ & 0.009 & -0.004 & -0.001 & -0.001 & -0.076 & 0.056 & -0.029 & 0.054 & -0.079 & 0.220 & -0.149 & 0.612 & $0.612 * *$ \\
\hline \multirow[t]{2}{*}{$\mathrm{X12}$} & $P$ & 0.000 & -0.005 & 0.005 & 0.020 & 0.080 & -0.051 & 0.014 & 0.005 & 0.020 & 0.001 & 0.014 & 0.804 & $0.771 * *$ \\
\hline & $G$ & -0.014 & 0.000 & 0.001 & -0.015 & -0.384 & 0.522 & -0.064 & -0.051 & -0.060 & 0.123 & -0.090 & 1.012 & 0.979** \\
\hline
\end{tabular}

Phenotypic residual effect $=0.061$ and genotypic residual effect $=0.039$. Values on main diagonal denotes direct effect

$\mathbf{X}_{\mathbf{1}}$ - Branches per plant

$\mathbf{X}_{2}$ - Days to $50 \%$ flowering

$\mathbf{X}_{\mathbf{3}}$ - Days to maturity

$\mathbf{X}_{4}$ - Plant height $(\mathrm{cm})$
$\mathbf{X}_{5}$ - Pegs per plant

$\mathbf{X}_{\mathbf{6}}$ - Pods per plant

$\mathbf{X}_{7}$ - Mature pods per plant

$\mathbf{X}_{\mathbf{8}}$ - Per cent pod set
$\mathbf{X}_{\mathbf{9}}$ - Harvest index

$\mathbf{X}_{\mathbf{1 0}}$ - Shelling percentage

$\mathbf{X}_{11}$ - 100-Kernel weight

$\mathbf{X}_{\mathbf{1 2}}$ - Pod yield per plant (g) 
Positive indirect effects of pod yield were exerted on kernel yield through days to maturity, plant height, pegs per plant, pods per plant, mature pods per plant, harvest index and 100-kernel weight. Korat et al., (2010) reports are akin with our present experimental results. Days to maturity, plant height, pegs per plant, mature pods per plant, percent pod set and pod yield per plant had its indirect contribution through pods per plant, while $50 \%$ flowering and shelling percentage exerted its negative indirect influence on kernel yield per plant. Similar reports of Khanpara et al., (2010) and Dhaliwal et al., (2010) were in line with our present findings. Pegs per plant manifested negative indirect influence through shelling percentage but harvest index and 100-kernel weight had positive indirect effect.

Per cent pod set exhibited positively indirect contribution on kernel yield through days to $50 \%$ flowering, days to maturity and harvest index, however, branches per plant, plant height, pods per plant, mature pods per plant and shelling percentage showed negative influence on kernel yield per plant. The characters pod yield per plant, shelling percentage, harvest index and 100-kernel weight had significant positive association with kernel yield and among themselves. Hence, simultaneous improvement of these traits will be more reliable for improving kernel yield and selecting improved genotypes of groundnut. In this study, residual effect was relatively very low $\left(R^{2}=\right.$ 0.061) indicating that adequate characters were utilized for the study.

It could be inferred from the present study that mature pods per plant, pod yield per plant, pods per plant, pegs per plant, harvest index, shelling percentage and 100-kernel weight are the major yield contributing characters in groundnut. Therefore, due emphasis should be given to these traits in formulating the criterion in the selection programmes to evolve high yielding genotypes of groundnut.

\section{References}

Alam, M.S., Rahman, A.R.M.S. and Khair, A.B.M.A. 1985. Genetic variability and character association in groundnut (Arachis hypogaea L.). Bangladesh J. Agric. Res., 10(4): 9-16.

Antony, E., Doddamani, M.B., Mummigatti, U.V. and Chetti, M.B. 2000. Correlation studies in groundnut (Arachis hypogaea L.) genotypes. Crop Res. Hisar, 19(3): 535-537.

Dewey, D.R. and Lu, K.H. 1959. A correlation and path coefficient analysis of components of crested wheat grass seed production. Agron. J., 51: 515-518.

Dhaliwal, G.P.S., Nagda, A.K. and Mittal, V.P. 2010. Inter-trait associations and path analysis studies in groundnut (Arachis hypogaea L.). Crop improv., 37(1): 57-60.

Giri, R.R., Toprope, V.N. and Jagtap, P.K. 2009. Genetic variability, character association and path analysis for yield, its component traits and late leaf spot, Phaeoisariopsis personata (Berk and curt) in groundnut. Int. J. Pl. Sci., 4: 551-555.

Islam, M.S. and Rasul, M.G. 1998. Genetic parameters correlation and path coefficient analysis in groundnut (Arachis hypogaea L.). Bangladesh J. Scient. Indus. Res., 33(2): 250-254.

Jain, S., Singh, P.B. and Sharma, P.P., 2016, Correlation and path analysis in groundnut (Arachis hypogaea L.), Int. J. Curr. Res., 8(08): 35811-35813.

Johnson, H.W., Robinson, H.F. and Comstock, R.E. 1955. Estimates of genetic and environmental variability in soybean. Agron. J., 47: 413-418.

Khanpara, M.D., Shinde, P.P., Jivani, L.L., 
Vachhani, J.H. and Kachhadia, V.H. 2010. Character association and path coefficient analysis in groundnut (Arachis hypogaea L.). Pl. Archives, 10(2): 695-698.

Korat, V.P., Pithia, M.S., Savaliya, J.J., Pansuriya, A. G. and Sodavadiya, P.R. 2010. Studies on characters association and path analysis for seed yield and its components in groundnut (Arachis hypogaea L.). Legume Res., 33(3): 211216.

Mahalakshmi, P., Manivannan, N. and Muralidharan, V. 2005. Variability and correlation studies in groundnut (Arachis hypogaea L.). Legume res., 28(3): 194-197.

Patil, K. G., Kenchanagoudar, P. V., Parameshwarappa, K. G. and Salimath, P. M. 2006. A study of correlation and path analysis in groundnut. Karnataka J. Agric. Sci., 19(2): 272-277.

Pavan Kumar, C., Rekha, R., Venkateswarulu, O. and Vasanthi, R.P. 2014. Correlation and path coefficient analysis in groundnut (Arachis hypogaea L.). Int. J. Appl. Biol. Pharma. Tech., 5(1): 8-11.

Prabhu, R., Manivannan, N., Mothilal., A. and Ibrahim, S.M. 2016. Studies on characters association for yield and its components in groundnut (Arachis hypogaea L.) Curr. Adv. Agric. Sci., 8(1): 49-54.

Rajarathinam, P., Narayana, M., Alagirisamy M. and Moshin, I.S., 2017, Variability, correlation and path coefficient analysis in groundnut (Arachis hypogaea L.). S. Vignesh and A. Philip Arokiadoss (ed.) Statistical Approaches on Multidisciplinary Research, Volume I, Surragh Publishers, India, 2017. DOI://doi.org/10.5281/zenodo.262967.

Ramakrishnan, P., Manivannan, N., Mothilal, A. and Mahalingam, L. 2017. Correlation studies in back cross derived population for foliar disease resistance in groundnut (Arachis hypgaea L.). Int. J. Curr. Microbiol. App. Sci., 6(5): 266-272.

Rasheed, B., Rasheed, H.A., Farid, A. and Khan, A. 2015. Characters association and path analysis of peanut (Arachis hypogaea L.) for pod yield. J. Agric. Sci. Res., 2(1): 79-84.

Sawargaonkar, S.L., Giri, R.R. and Hudge, B.V. 2010. Character association and path analysis of yield component traits and late leaf spot disease traits in groundnut (Arachis hypogaea L.). Agric. Sci. Digest, 30(2): 115-119.

Shashi Kumara, P., Sanjeev, B.G. and Venkata Ravana, P. 2015. Studies on correlation and path analysis for traits related to water use efficiency and pod yield and its components in groundnut (Arachis hypogaea L.). The Bioscan, 10(4): 2155-2158.

Shoba, D., Manivannan, N. and Vindhiyavarman, P. 2012. Correlation and path coefficient analysis in groundnut (Arachis hypogaea L.). Madras Agric. J., 99(1-3): 18-20.

Shukla, A.K. and Kumar Rai, P. 2014. Evaluation of groundnut genotypes for yield and quality traits. Ann. Pl. Soil Res., 16(1):41-44.

Sumathi, P. and Muralidharan, V. 2007. Character association and path coefficient analysis in confectionery type groundnut (Arachis hypogaea $\mathrm{L}$.). Madras Agric. J., 94(1/6): 105-109.

Thirumala Rao, V., 2016, Genetic variability, correlation and path coefficient analysis under drought in groundnut (Arachis hypogaea L.). Legume Res., 39(1): 319322.

Vaithiyalingan, V., Manoharan and Ramamoorthi, N. 2010. Association analysis among the yield and yield attributes of early season drought tolerant groundnut (Arachis hypogaea 
L.). Electronic J. Pl. Breed., 1(5): 13471350.

Vange, T. and Maga, T.J. 2014. Genetic characteristics and path coefficient analysis in ten groundnut varieties (Arachis hypogaea L.) evaluated in the Guinea Savannah agro-ecological zone. African J. Agric. Res., 9(25): 1932-
1937.

Vijayasekhar, 2002. Genetic divergence studies through $\mathrm{D}^{2}$ statistic and isozyme analysis in Spanish bunch groundnut (Arachis hypogaea L.) M.Sc. (Ag.). Thesis submitted to Acharya N.G. Ranga Agricultural University, Hyderabad.

\section{How to cite this article:}

Trivikrama Reddy, A., M. Reddi Sekhar, A. Vijayabharathi, T. Lakshmi Pathy, G. Lakshmikantha Reddy and Jayalakshmi, V. 2017. Correlation and Path Analysis of Kernel Yield and its Components in Groundnut (Arachis hypogaea L.). Int.J.Curr.Microbiol.App.Sci. 6(12): 10-16. doi: https://doi.org/10.20546/ijcmas.2017.612.002 